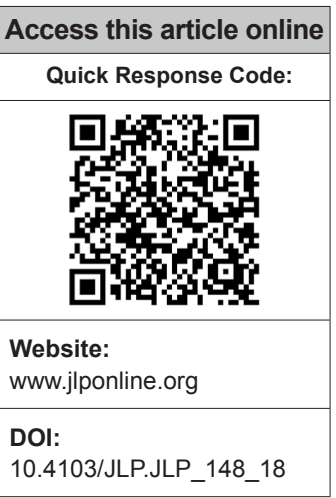

Vector Control Research Centre, Puducherry,

${ }^{2}$ Department of Microbiology, Pondicherry Institute of Medical Sciences, Puducherry, ${ }^{1}$ Regional Medical Research Centre, Belagavi, Karnataka, India

Address for correspondence:

Dr. Sugeerappa

Laxmanappa Hoti, Regional Medical

Research Centre, Nehru Nagar, Belagavi,

Karnataka, India.

E-mail: slhoti@yahoo.com

Submission: $30-11-2018$ Accepted: 22-01-2019

\section{Evaluation of loop-mediated isothermal amplification assay for detection of scrub typhus in patients with acute febrile illness presenting to a Tertiary Care Center in Puducherry, India}

\author{
Patricia Anitha Karthikeyan, Sugeerappa Laxmanappa Hoti ${ }^{1}$, Reba Kanungo $^{2}$
}

\begin{abstract}
:
PURPOSE: Scrub typhus an acute febrile illness has diverse clinical manifestations, which overlap with other febrile illnesses. Due to this reason, it is misdiagnosed, leading to inappropriate treatment, sometimes resulting in fatality. Thus, accurate diagnosis of scrub typhus is important for appropriate treatment. This study evaluated the loop-mediated isothermal amplification (LAMP) assay as a diagnostic test for scrub typhus among patients with fever.
\end{abstract}

MATERIALS AND METHODS: A total of 50 cases of acute febrile illness clinically resembling scrub typhus, with or without an eschar, or cases of pyrexia of unknown origin were included in the study. Blood samples collected from these cases were subjected to detection of IgM antibodies to Orientia tsutsugamushi by ELISA, conventional groEL polymerase chain reaction (PCR), and the LAMP assay.

RESULTS: Twelve cases had fever for less than a week, and two had fever for more than 3 weeks. IgM antibodies to 0 . tsutsugamushi were detected in 37 out of 50 samples (74\%). LAMP assay was positive in 33 samples (66\%). groEL gene-based PCR detected 35 (70\%) samples as positive. Two samples negative by LAMP assay were positive by this PCR. Twenty samples collected from patients with dengue, typhoid, and malaria tested by the LAMP assay were negative, indicating its good specificity. LAMP assay and the conventional groEL-based PCR could detect $72.7 \%$ and $74.3 \%$ of the samples, respectively before the $10^{\text {th }}$ day after onset of fever, whereas IgM ELISA could detect only $40.5 \%$ of the 37 samples.

CONCLUSION: This study suggests that LAMP assay could be a useful diagnostic test for detecting scrub typhus in the acute phase of the illness and a cheaper alternative to other molecular methods in resource poor settings.

Key words:

Diagnosis, groEl polymerase chain reaction, loop-mediated isothermal amplification assay, scrub typhus

\section{Introduction}

Crub typhus, caused by Orientia Stsutsugamushi and transmitted by the bite of chiggers of Leptotrombidium deliense, is an acute febrile illness. ${ }^{[1,2]}$ This disease is endemic in the so-called "tsustugamushi

This is an open access journal, and articles are distributed under the terms of the Creative Commons Attribution-NonCommercial-ShareAlike 4.0 License, which allows others to remix, tweak, and build upon the work non-commercially, as long as appropriate credit is given and the new creations are licensed under the identical terms.

For reprints contact: reprints@medknow.com triangle" but has been recently reported from many areas beyond this. ${ }^{[3,4]}$ It is also being increasingly reported from various parts of India, in recent years. ${ }^{[5-7]}$ In Puducherry, the disease has been reported from several areas which includes a case series involving meningitis among scrub typhus cases. $[5,6,8-10]$ One of the studies from this region

How to cite this article: Karthikeyan PA, Hoti SL, Kanungo R. Evaluation of loop-mediated isothermal amplification assay for detection of scrub typhus in patients with acute febrile illness presenting to a Tertiary Care Center in Puducherry, India. J Lab Physicians 2019;11:82-6. 
estimated the prevalence of the disease among cases with fever to be around $22 \%{ }^{[11]}$ Although the clinical presentation of cases with scrub typhus is usually, only an undifferentiated fever, it may resemble typhoid, leptospirosis, and/or dengue. The presence of an eschar can differentiate scrub typhus from other fevers, but its presence is highly variable $(7 \%-68 \%)$. Complications due to this disease include meningitis, pneumonitis, acute respiratory distress syndrome, acute renal failure, hypotensive shock, multiorgan failure, and disseminated intravascular coagulation. ${ }^{[12,13]}$ Mortality due to scrub typhus can be as high as $36.8 \%$ in some endemic areas. ${ }^{[14]}$

Since the clinical manifestations of scrub typhus overlap with other febrile illnesses, it is misdiagnosed, leading to inappropriate treatment, which may result in fatality. Thus, accurate diagnosis of the disease is very important for proper case treatment, as administration of doxycycline in these cases could be life-saving. ${ }^{[15]}$ In the past, the diagnosis of this infection was mainly based on clinical features which are nonspecific. In some instances, Weil-Felix test was used for supporting the clinical diagnosis, but this test, though specific, has poor sensitivity. ${ }^{[16]}$ Immunofluorescence assay considered the gold standard test for the diagnosis of scrub typhus, is expensive and not readily available in India. Polymerase chain reaction (PCR) assay using specific primers targeting genes coding for $56 \mathrm{kDa}, 47 \mathrm{kDa}$ or groEl proteins by real-time methods have been used recently. ${ }^{[2,17]}$ However, the cost and expertise required, make real-time-based methods impractical in resource poor settings. Recently, a simpler version of molecular assay, Loop-mediated isothermal amplification (LAMP) assay has been developed. It is a novel method of isothermal real-time gene amplification and detection, which is rapid and cost-effective requiring only a water bath or heating block instead of a thermocycler and hence can be a Point-of-Care diagnostic. LAMP assay has been used in recent years, for the rapid detection of several infectious diseases such as human African trypanosomiasis, dengue, malaria due to Plasmodium vivax and tuberculosis. ${ }^{[18-21]}$

This method, being simple and less expensive, has the potential for application in remote areas, both as a diagnostic and epidemiological tool. Paris et al. have developed a LAMP assay for detection of O. tsutsugamushi which was reported to have high accuracy. ${ }^{[22]}$ The present study evaluates this LAMP assay as a diagnostic test for scrub typhus in Puducherry, an area endemic for the infection.

\section{Materials and Methods}

This study was approved by the Institutional Ethics Committee of Pondicherry Institute of Medical Sciences, a tertiary care hospital in Puducherry, India. A total of 50 blood samples were collected from patients who had fever for more than 5 days, after obtaining informed and written consent. The inclusion criteria were patients with fever whose cause was not known (pyrexia of unknown origin), or those who had fever clinically resembling scrub typhus, with or without an eschar and exclusion criteria were patients who tested positive for infections such as dengue, malaria, leptospirosis, and typhoid fever. A suspected or a clinical case of scrub typhus is one who has an acute febrile illness for 5 days or more, with or without an eschar. Other features included headache, rash, lymphadenopathy, and multiorgan involvement. ${ }^{[23]}$ Dengue was tested by Dengue day 1 test (J. Mitra, India). Malaria was ruled out by microscopic examination of stained peripheral blood smear and Parascreen Rapid test for Malaria (Zephyr Biomedicals, India), leptospirosis was detected by Leptospira IgM and IgG card test (SD Bioline, India). Typhoid was detected by conventional blood culture and Widal test, which was done using stained Salmonella antigens (Span, India). Serum was separated from these samples, and IgM ELISA was performed to detect antibodies against $O$. tsutsugamushi using Scrub Typhus Detect IgM ELISA system (InBios International Inc., USA) as per the manufacturer's instructions. The cutoff was determined, as 0.406 , calculated based on optical density (O. D) values obtained after performing IgM ELISA on 300 samples, which included known scrub typhus positive samples, sera from normal individuals, and sera from patients with other fevers. A relative operating characteristic curve was drawn to arrive at the cutoff (unpublished data). Samples with O. D value more than the cutoff were considered positive for $O$. tsutsugamushi-specific IgM antibodies. Demographic parameters of the cases were also recorded in a pro forma.

\section{LAMP Assay}

The whole genomic DNA was extracted from blood samples using blood genomic extraction kit as per the manufacturer's instructions (Sigma-Aldrich, USA). LAMP reaction was set up on the extracted DNA. The primers for groEl gene used for the reaction were those described by Paris et al. ${ }^{[22]}$ and was synthesized from Eurofins, Bengaluru, India. The details of primers are as follows:

F3 5' - TG A C CGYGGA T A T A T A TCACA - 3' B3 5'-CAATGCRGTAAGAGCTTCTC-3' FIP (F1c-F2) 5' - GC A C T G T A GA T A C C T TC T GA T C C A A T ACTTTGCA A C R A A T C GTGA A - 3' B I P (B1C-B2) 5'-CCACTKGTTCCTGTGCTTGAC G T C T A C A T C A T C A GC A A T C A - 3' LF 5'-GGATTTTCAAATTCRGTAATCATCTT-3' LB 5'-CTCAYACTGGCAAGCCATTA-3'. 
The reaction mixture contained $16 \mathrm{mM} \mathrm{MgSO}_{4^{\prime}} 1.6 \mathrm{M}$ Betaine, $2.8 \mathrm{mM}$ each of dNTP, $20 \mathrm{mM} \mathrm{NH}_{4} \mathrm{SO}_{4}, 3 \mu \mathrm{l}$ primers (5 pmol of F3 and B3, 20 pmol of Loop-F and Loop-B, and 40 pmol of FIP and BIP), $4 \mu \mathrm{l}$ of DNA, and $1 \mu \mathrm{l}$ of Bst DNA polymerase in a $30 \mu \mathrm{l}$ reaction. This was incubated in a water bath at $63^{\circ} \mathrm{C}$ for $90 \mathrm{~min}$. The reaction was subsequently terminated by holding the tubes at $80^{\circ} \mathrm{C}$ for $10 \mathrm{~min}$. Each set of LAMP reaction was set up in duplicate. One of the tubes contained $120 \mu \mathrm{mol}$ hydroxy naphthol blue (HNB) as an indicator, and the other tube did not contain any dye. The interpretation of end-point in LAMP assay was by detection of change in color from light blue to deep blue to purple in the tube with HNB and turbidity in the tube without the dye. All the amplified LAMP products were simultaneously electrophoresed on $2 \%$ agarose gel to compare the positivity by the presence of ladder-like bands.

\section{Detection of gene encoding groEL by conventional polymerase chain reaction}

The primers used in this PCR were described earlier by Patricia et al. ${ }^{[2]}$ The primer sequence is as follows:

groEL F: 5'- TTGCTGATGATGTAGACGGA- 3'and groEL R: 5'- TGTTCACAACGAGAATTAACTT- ${ }^{\prime}$.

These primers were designed to amplify a $300 \mathrm{bp}$ product. The reaction mixture contained Green Master Mix (Promega, USA), $10 \mathrm{pmol}$ each of forward and reverse primers and $4 \mu \mathrm{l}(50 \mathrm{ng} / \mathrm{ml})$ of extracted DNA in a final volume of $30 \mu \mathrm{l}$. The cycling conditions were $95^{\circ} \mathrm{C}$ for $20 \mathrm{~s}, 52^{\circ} \mathrm{C}$ for $45 \mathrm{~s}$ and $72^{\circ} \mathrm{C}$ for $1 \mathrm{~min}$, repeated 30 times in a Master Cycler Gradient (Eppendorf, Germany). Amplicons from a positive sample were subjected to nucleic acid sequencing and confirmed as that of the groEl gene of Orientia and were used as positive control for further tests.

\section{Results}

Fifty blood samples collected from suspected cases of scrub typhus were subjected to scrub typhus specific, IgM antibody detection by ELISA, conventional groEL PCR, and the LAMP assays. These cases came from various urban and rural areas of Puducherry and areas of Tamil Nadu state. However, coastal regions of Puducherry and Tamil Nadu located on the eastern coast of the country such as Kalapet, Pillaichavady, and Bommaiyarpalayam had more number of cases. There was preponderance in adults, who comprised $76 \%$ of the cases and almost equal distribution among males and females, the proportion being 27:23. Most number of cases was in the 25-34 age group (i.e.,) $26 \%$ of them. This study found a seasonal trend with an increase in the number of cases from September which marks the end of monsoon in this region, and also in the cooler months of December and January. Cases declined in the hot season.

IgM antibodies to O. tsutsugamushi were detected in 37 out of 50 samples $(74 \%)$. The remaining 13 samples were negative for Orientia antibodies and also for other common febrile illnesses in the region. LAMP assay was positive in 33 samples (66\%). There was a concordance between different methods of interpretation of the LAMP test. All positive samples had a pellet at the bottom of the tube, a color change from light blue to purple and gel electrophoresis of amplicons of the assay had ladder patterns. Samples that were negative had no pellet, did not show any color change and had no ladder patterns on gel electrophoresis. The color change in tubes with HNB dye is shown in Figure 1. These samples were also subjected to PCR to detect the groEL gene, which was positive in $35(70 \%)$ samples [Figure 2]. Two samples which were negative by LAMP assay were positive by this PCR.

To test the occurrence of false positives by the LAMP assay if any, DNA was extracted from 20 samples collected from patients with dengue, typhoid, and malaria. All these samples were negative, indicating the high specificity of the assay.

Among the 50 cases, 12 had an acute febrile illness (i.e.) fever for less than week and 2 had a fever for more than 3 weeks. An analysis was done to associate the positivity of the three assays, i. e., ELISA, LAMP assay, and groEL PCR with the time of fever in these cases is shown in Table 1 . The positivity of samples in the three tests was $37(74 \%)$ by ELISA, $33(66 \%)$ by LAMP assay, and $35(70 \%)$ by groEL PCR. The IgM ELISA detected $2(4 \%)$ samples as positive up to 7 days of onset of fever and $13(26 \%)$ samples between 7 and 10 days of fever. The positivity of samples significantly increased after 10 days and $22(44 \%)$ samples were positive [Table 1]. Both the molecular assays detected a higher number, i.e., $11(22 \%)$ by LAMP assay and 12 by groEl PCR $(24 \%)$ as positive within 7 days of onset of fever. The LAMP assay

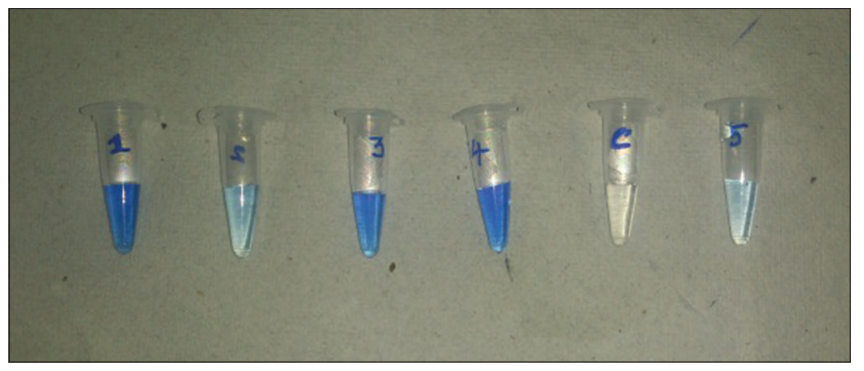

Figure 1: End-point of loop-mediated isothermal amplification assay using hydroxy naphthol blue. Tubes 1,3 and 4 show deepening of blue color indicating positive reaction and Tubes 2 (negative sample) and 6 (negative control) with light blue color indicate negative reaction. Tube 5 contains water to show the colour difference 


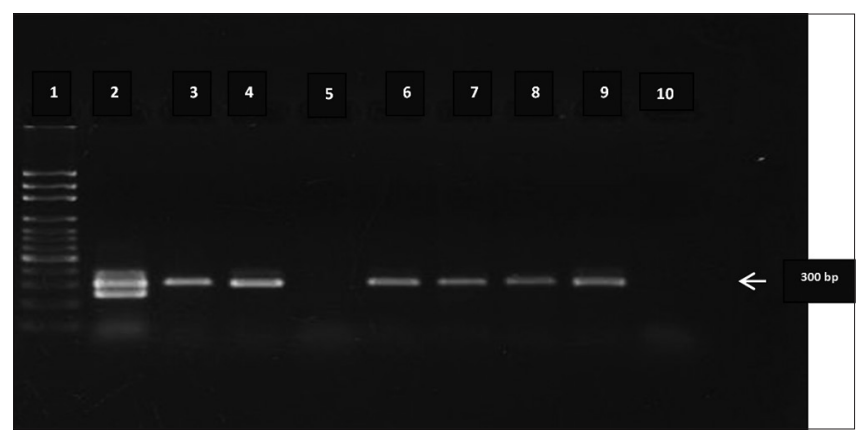

Figure 2: Gel electrophoresis picture of amplicons obtained by groEL polymerase chain reaction. Lane $1-100$ bp ladder, Lane 9 - positive control and Lane 10 - negative control. Lanes 2, 3, 4, 6, 7 and 8 show amplification products in the polymerase chain reaction while Lane 5 (suspected case) shows sample with no amplification

detected 13 (26\%) samples while groEL PCR detected $14(28 \%)$ samples as positive between $7^{\text {th }}$ and $10^{\text {th }}$ day postfever By the $10^{\text {th }}$ day of fever, the assays could detect $9(18 \%)$ samples as positive further. Thus, the two molecular assays, the LAMP assay, and the conventional groEL-based PCR assay could detect $72.7 \%$ and $74.3 \%$ of the samples as positive, respectively, before 10 days after onset of fever, whereas IgM ELISA could detect only $40.5 \%$ of the 37 samples.

\section{Discussion}

Scrub typhus, which manifests as an acute febrile illness is being reported in increasing numbers from the study area. Sensitive, rapid, cheap, and simple tests are required to detect this infection, which predominantly occurs in resource-poor endemic areas. LAMP assay, described in recent years is a simple and rapid method of diagnosis of infectious diseases. The results of the test can be made available in a shorter time of about 90 min, unlike conventional or real-time PCR assays. It can be performed with only a heating block and does not require a thermocycler. The results of this assay can be read by visual discrimination using several dyes for the visual detection of end-point of LAMP reaction. The dyes include calcein (either alone or in combination with $\mathrm{MnCl}_{2}$ ), SYBR Green I, Propidium iodide, Quant-iT Picogreen, and HNB. HNB is the cheapest dye among the indicators and found to be the most reliable ${ }^{[25]}$ and hence was used in this study. Hence, the assay can be conveniently used for the diagnosis of infectious diseases in endemic areas. LAMP assays thus have the potential to fulfill the WHO "ASSURED" criteria (affordable, sensitive, specific, user-friendly, robust and rapid, equipment free, deliverable to those who need them) for diagnostic methodologies, as they can detect pathogen-derived nucleic acid with low technical expenditure. ${ }^{[26]}$

The recently developed LAMP assay for the detection of scrub typhus is reported to be about $53 \%$ sensitive
Table 1: Association between duration of fever and positivity of three tests to detect scrub typhus

\begin{tabular}{lcccc}
\hline \multirow{2}{*}{ Test } & \multicolumn{4}{c}{ Time of fever (number of samples positive) } \\
\cline { 2 - 5 } & $<\mathbf{7}$ days & $\mathbf{7 - 1 0}$ days & $>10$ days & Total \\
\hline ELISA IgM & 2 & 13 & 22 & 37 \\
LAMP assay & 11 & 13 & 9 & 33 \\
groEL PCR & 12 & 14 & 9 & 35 \\
\hline
\end{tabular}

${ }^{*}$ From 10 to a maximum of 14 days. ELISA=Enzyme-linked immunosorbent assay, IgM=Immunoglobulin M, LAMP=Loop-mediated isothermal amplification, $\mathrm{PCR}=$ Polymerase chain reaction

and $94 \%$ specific. The assay is based on the groEL gene of $O$. tsutsugamushi, which is highly conserved among these organisms and has been found to be highly specific, based on the conventional PCR assay. ${ }^{[27]}$ In the present study, hence, we selected the groEL gene-based LAMP assay for the detection of $O$. tsutsugamushi for the evaluation of its diagnostic accuracy. The evaluation was done in 50 cases confirmed to be of scrub typhus based on the clinical picture and the results of a serological test, namely ELISA, which detects IgM antibodies against Orientia with a sensitivity of $100 \%$ and specificity of $73 \% .^{[28]}$

Of 50 suspected cases of scrub typhus, 33 (66\%) were positive by LAMP assay while $37(74 \%)$ and $35(70 \%)$ samples were positive by IgM ELISA and groEL PCR, respectively. Since the gold standard for detection of the disease, i.e., microimmunofluorescence was not employed in the study the sensitivity and specificity of the molecular tests was compared with ELISA and LAMP was found to be $89 \%$ sensitive and $100 \%$ specific with an accuracy of $92 \%$ while groEl PCR was found to be $94.5 \%$ sensitive and $100 \%$ specific with an accuracy of $96 \%$. The lower rate of detection of cases by LAMP assay compared to IgM ELISA could be because 17 LAMP assay negative samples, were from patients who had fever for more than 10 days, and were treated with doxycycline empirically, which would have cleared the bacterial load. As the bacterial load decreased with antibiotic therapy, LAMP assay may have been unable to pick up the DNA as the threshold for the assay ranges from 14 to 44 copies / $\mu l^{[22]}$ In an earlier study, which used the STIC criteria as the reference comparator, the scrub typhus LAMP assay gave a sensitivity of $52 \%$ (95\% confidence interval [CI], $38-66)$ and specificity of $94 \%(95 \% \mathrm{CI}, 88-98) .{ }^{[29]}$ However, in the present study, LAMP assay was positive in 11 patients, whose blood was collected during the $1^{\text {st }}$ week of fever with the exception of 1 additional sample, which was positive by conventional groEL PCR. The present study supports earlier reports of low LAMP positivity after 10 days of fever, as compared to IgM detection, which continued to rise after this period, detecting $66 \%$ of the suspected cases. ${ }^{[27]}$ All these patients were treated with Doxycycline (100 mg bd for a week) or Azithromycin (500 mg qd for 5 days) and recovered completely. Although this study was done on a limited 
number of samples, it reiterates the importance of this rapid and cost-effective assay in areas of endemicity, particularly in the early phase of fever.

\section{Conclusion}

Scrub typhus can manifest with many atypical presentations and if not diagnosed properly, would lead to either inappropriate treatment or severe complications/fatality. Easy and rapid methods of laboratory diagnosis are required in endemic areas for imparting necessary treatment and saving the lives of infected patients, who might have fatal consequence if not treated properly. This study suggests that LAMP assay could be a useful diagnostic test for detecting scrub typhus in the acute phase of the illness and a cheaper alternative to other molecular methods in resource poor settings.

\section{Financial support and sponsorship}

This study was funded by the Indian Council of Medical Research as part of intramural research funding for Vector Control Research Centre (Puducherry) Grant Sanction Number: IM 1204.

\section{Conflicts of interest}

There are no conflicts of interest.

\section{References}

1. Watt G, Parola P. Scrub typhus and tropical rickettsioses. Curr Opin Infect Dis 2003;16:429-36.

2. Koh GC, Maude RJ, Paris DH, Newton PN, Blacksell SD. Diagnosis of scrub typhus. Am J Trop Med Hyg 2010;82:368-70.

3. Izzard L, Fuller A, Blacksell SD, Paris DH, Richards AL, Aukkanit $\mathrm{N}$, et al. Isolation of a novel Orientia species (O. chuto sp. Nov.) from a patient infected in Dubai. J Clin Microbiol 2010;48:4404-9.

4. Maina AN, Farris CM, Odhiambo A, Jiang J, Laktabai J, Armstrong J, et al. Q fever, scrub typhus, and Rickettsial diseases in Children, Kenya, 2011-2012. Emerg Infect Dis 2016;22:883-6.

5. Vivekanandan M, Mani A, Priya YS, Singh AP, Jayakumar S, Purty S, et al. Outbreak of scrub typhus in Pondicherry. J Assoc Physicians India 2010;58:24-8.

6. Stephen S, Sangeetha B, Ambroise S, Sarangapani K, Gunasekaran D, Hanifah M, et al. Outbreak of scrub typhus in Puducherry \& Tamil Nadu during cooler months. Indian J Med Res 2015;142:591-7.

7. Arun Babu T, Vijayadevagaran V, Ananthakrishnan S. Characteristics of pediatric scrub typhus eschar in South Indian children. Pediatr Dermatol 2017;34:124-7.

8. Prabagaravarthanan R, Harish BN, Parija SC. Typhus fever in Pondicherry. J Commun Dis 2008;40:159-60.

9. Anitharaj V, Stephen S, Pradeep J, Park S, Kim SH, Kim YJ, et al. Serological diagnosis of acute scrub typhus in Southern India: Evaluation of InBios scrub typhus detect IgM rapid test and comparison with other serological tests. J Clin Diagn Res 2016;10:DC07-10.

10. Viswanathan S, Muthu V, Iqbal N, Remalayam B, George T. Scrub typhus meningitis in South India - A retrospective study. PLoS

\section{One 2013;8:e66595.}

11. Roopa KS, Karthika K, Sugumar M, Bammigatti C, Shamanna SB, Harish BN, et al. Serodiagnosis of scrub typhus at a tertiary care hospital from Southern India. J Clin Diagn Res 2015;9:DC05-7.

12. Kawamura A Jr., Tanaka H. Rickettsiosis in Japan. Jpn J Exp Med 1988;58:169-84.

13. Sirisanthana V, Puthanakit T, Sirisanthana T. Epidemiologic, clinical and laboratory features of scrub typhus in thirty Thai children. Pediatr Infect Dis J 2003;22:341-5.

14. Narvencar KP, Rodrigues S, Nevrekar RP, Dias L, Dias A, Vaz M, et al. Scrub typhus in patients reporting with acute febrile illness at a tertiary health care institution in Goa. Indian J Med Res 2012;136:1020-4.

15. Walker DH. Scrub typhus - Scientific neglect, ever-widening impact. N Engl J Med 2016;375:913-5.

16. Isaac R, Varghese GM, Mathai E, Manjula J, Joseph I. Scrub typhus: Prevalence and diagnostic issues in rural Southern India. Clin Infect Dis 2004;39:1395-6.

17. Batra HV. Spotted fevers \& typhus fever in Tamil Nadu. Indian J Med Res 2007;126:101-3.

18. Wastling SL, Picozzi K, Kakembo AS, Welburn SC. LAMP for human African trypanosomiasis: A comparative study of detection formats. PLoS Negl Trop Dis 2010;4:e865.

19. Teoh BT, Sam SS, Tan KK, Johari J, Danlami MB, Hooi PS, et al. Detection of dengue viruses using reverse transcription-loop-mediated isothermal amplification. BMC Infect Dis 2013;13:387.

20. Patel JC, Oberstaller J, Xayavong M, Narayanan J, DeBarry JD, Srinivasamoorthy G, et al. Real-time loop-mediated isothermal amplification (RealAmp) for the species-specific identification of Plasmodium vivax. PLoS One 2013;8:e54986.

21. Iwamoto T, Sonobe T, Hayashi K. Loop-mediated isothermal amplification for direct detection of Mycobacterium tuberculosis complex, M. avium, and M. intracellulare in sputum samples. J Clin Microbiol 2003;41:2616-22.

22. Paris DH, Blacksell SD, Newton PN, Day NP. Simple, rapid and sensitive detection of Orientia tsutsugamushi by loop-isothermal DNA amplification. Trans R Soc Trop Med Hyg 2008;102:1239-46.

23. Rahi M, Gupte MD, Bhargava A, Varghese GM, Arora R. DHR-ICMR guidelines for diagnosis \& management of Rickettsial diseases in India. Indian J Med Res 2015;141:417-22.

24. Patricia KA, Hoti SL, Kanungo R, Jambulingam P, Shashikala N, Naik AC, et al. Improving the diagnosis of scrub typhus by combining groEL based polymerase chain reaction and $\operatorname{IgM}$ ELISA. J Clin Diagn Res 2017;11:DC27-31.

25. Goto M, Honda E, Ogura A, Nomoto A, Hanaki K. Colorimetric detection of loop-mediated isothermal amplification reaction by using hydroxy naphthol blue. Biotechniques 2009;46:167-72.

26. Njiru ZK. Loop-mediated isothermal amplification technology: Towards point of care diagnostics. PLoS Negl Trop Dis 2012;6:e1572.

27. Paris DH, Blacksell SD, Nawtaisong P, Jenjaroen K, Teeraratkul A, Chierakul W, et al. Diagnostic accuracy of a loop-mediated isothermal PCR assay for detection of Orientia tsutsugamushi during acute scrub typhus infection. PLoS Negl Trop Dis 2011;5:e1307.

28. Prakash JA, Kavitha ML, Mathai E. Nested polymerase chain reaction on blood clots for gene encoding $56 \mathrm{kDa}$ antigen and serology for the diagnosis of scrub typhus. Indian J Med Microbiol 2011;29:47-50.

29. Blacksell SD, Paris DH, Chierakul W, Wuthiekanun V, Teeratakul A, Kantipong P, et al. Prospective evaluation of commercial antibody-based rapid tests in combination with a loop-mediated isothermal amplification PCR assay for detection of Orientia tsutsugamushi during the acute phase of scrub typhus infection. Clin Vaccine Immunol 2012;19:391-5. 